\title{
Is isolated aortic valve replacement sufficient to treat concomitant moderate functional mitral regurgitation? A propensity-matched analysis
}

\author{
Robert A. Sorabella, Anna Olds, Halit Yerebakan, Dua Hassan, Michael A. Borger, Michael Argenziano, \\ Craig R. Smith and Isaac George* (iD
}

\begin{abstract}
Background: A significant proportion of patients presenting for isolated aortic valve replacement (AVR) demonstrate some degree of functional mitral regurgitation (fMR). Guidelines addressing concomitant mitral valve intervention in those patients with moderate fMR lack strong evidence-based support. Our aim is to determine the effect of untreated moderate fMR at the time of AVR on long-term survival.

Methods: All patients undergoing isolated AVR from 2000 to 2013 at our institution were retrospectively reviewed. Patients were stratified according to severity of preoperative fMR; 0-1+ MR (Group NoMR, $n=1826$ ) and 2-3+ MR (Group MR, $n=330$ ). All patients in Group MR were propensity-matched with patients in Group NoMR to control for differences in baseline characteristics. The primary outcome of interest was overall survival.

Results: Propensity analysis matched 330 patients from each group. Mean age was $77.9 \pm 10.0$ years and $50.6 \%$ were male. There were no differences in baseline demographics, echocardiographic parameters, or co-morbidities between groups. Kaplan-Meier analysis showed significantly worse medium and long-term survival in Group MR compared to Group NoMR (log-rank $p=0.02$ ). Follow-up echocardiography showed slightly more severe MR in Group MR (1.1 \pm 0.7 MR vs. $0.8 \pm 0.7$ NoMR, $p=0.03)$ at 1 year.

Conclusions: Patients undergoing isolated AVR with concomitant 2-3+ fMR experience poorer long-term survival than those patients with no or mild fMR. This suggests that mitral valve intervention may be necessary in patients undergoing AVR with clinically significant fMR.
\end{abstract}

Keywords: Aortic valve replacement, Mitral valve disease, Mitral regurgitation

\section{Background}

Surgical aortic valve replacement (AVR) remains the most common valvular operation with over 50,000 procedures performed in the United States in 2013 alone [1]. In addition to correction of primary aortic valve pathology, current American Heart Association guidelines recommend mitral valve intervention for patients presenting with concomitant severe functional mitral regurgitation (fMR) as a result of their aortic valve disease

\footnotetext{
* Correspondence: isaacgeorge@hotmail.com

Division of Cardiothoracic Surgery, New York Presbyterian Hospital -

Columbia University College of Physicians and Surgeons, 177 Fort

Washington Ave, MHB 7GN-435, New York, NY 10032, USA
}

[2-4]. However, according to published literature, up to two-thirds of patients with aortic stenosis or insufficiency (AS, AI, respectively) can present with moderate or less $\mathrm{AMR}$, and significant debate persists on whether these patients warrant mitral valve repair at the time of AVR [5-12].

The traditional, conservative perspective assumes that non-severe fMR regresses following isolated AVR due to left ventricular reverse remodeling and removal of afterload obstruction, which subsequently leads to improved mitral leaflet coaptation. Although this phenomenon occurs to some extent following AVR, some reports suggest that patients may still be left with clinically

(C) The Author(s). 2018 Open Access This article is distributed under the terms of the Creative Commons Attribution 4.0 International License (http://creativecommons.org/licenses/by/4.0/), which permits unrestricted use, distribution, and 
significant fMR even at late follow-up, which may lead to worsened long-term survival [13-15]. However, these studies are limited by small sample sizes and lack of extended follow-up, and fail to yield definitive conclusions. Therefore, the aim of this study is to determine the impact of uncorrected moderate fMR at the time of AVR on late survival in comparison to patients with no or mild fMR in a large patient population. These results may have important clinical implications in defining the appropriate treatment strategy for patients with combined aortic and moderate functional mitral valve disease, particularly in the modern era of transcatheter valve therapy.

\section{Methods}

\section{Patient selection}

All patients undergoing isolated AVR at NY Presbyterian-Columbia University Medical Center between January 2000 and December 2013 were retrospectively reviewed for inclusion into the study. Patients with severe fMR $(4+)$, primary mitral valve disease, prior mitral surgery, or cardiogenic shock at the time of surgery were excluded from the analysis. A total of 2156 patients met inclusion and exclusion criteria, and patients were stratified according to degree of preoperative $\mathrm{fMR}$ : $0-1+$ MR (Group NoMR, $n=1826)$ and $2-3+$ MR (MR, $n=330$ ). Functional MR was defined as MR with normal mitral valve morphology regardless of severity of left ventricular dysfunction as evaluated by preoperative transthoracic or transesophageal echocardiography. Degree of MR was assessed using a $0-4+$ scale, as graded by a blinded echocardiographer $(0=$ no MR, $1+=$ mild MR, $2+=$ moderate MR, $3+=$ moderate-severe MR, $4+=$ severe MR). Given the significant underlying differences in demographics and co-morbid conditions between groups, a propensity-matched analysis was performed using a subset of the overall cohort. The study was approved by the Columbia University Institutional Review Board and need for individual patient consent was waived.

Clinical and follow-up data were collected from the electronic medical record and mortality data for patients lost to follow up were collected from United States Social Security Death Index. Baseline demographics, co-morbidities [congestive heart failure, prior myocardial infarct, severe chronic kidney disease (eGFR $<30 \mathrm{~mL} / \mathrm{min}$ ), diabetes mellitus, end-stage renal disease needing dialysis, cerebrovascular disease, peripheral vascular disease, and chronic obstructive pulmonary disease], preoperative echocardiographic measurements, operative details, postoperative complications and length of stay, follow-up echocardiographic data, and survival data were collected for analysis.

\section{Statistical analysis}

All analyses were conducted using SPSS version 22 (IBM corporation, Armonk, NY). Continuous variables are presented as mean \pm standard deviation and compared using independent samples t-tests, or median and interquartile range and compared using Mann-Whitney $U$ test where appropriate. Categorical variables are presented as total count and percentage of the group, and compared using Pearson's chi-square test or Fisher's exact test where applicable. Kaplan-Meier analysis was used for comparison of survival, and survival curves were compared using the log-rank test. In order to control for differences in preoperative variables between groups, a propensity-matched analysis was performed. Patients were assigned a propensity score and matched using the nearest neighbor Greedy 5 to 1 digit matching algorithm (MatchIt package in R 3.0.2, R foundation for Statistical Computing, Vienna, Austria). Covariates included in calculation of the propensity score included age at surgery, gender, body mass index, preoperative ejection fraction, preoperative tricuspid regurgitation, preoperative hemoglobin, indication for surgery, and history of severe chronic kidney disease, cerebrovascular disease, myocardial infarction, peripheral vascular disease, chronic obstructive pulmonary disease, diabetes mellitus, need for hemodialysis, congestive heart failure, or prior cardiac surgery. Matching was done in a 1:1 fashion and matched 330 patients from each group for comparison. All $p$-values $\leq 0.05$ were considered statistically significant.

\section{Results}

\section{Overall cohort analysis}

Baseline characteristics, operative details, and outcomes of the overall cohort analysis are presented in Table 1. Patients in Group MR were significantly older, had lower BMIs, and generally had more preoperative co-morbidities than those in Group NoMR. Preoperative echocardiograms revealed significantly lower left ventricular ejection fractions and higher rates of severe aortic stenosis (AS) and severe tricuspid regurgitation (TR) in Group MR. Analysis of postoperative complications showed that patients in Group MR had a significantly higher 30 -day mortality rate $(3.6 \%$ MR vs. $1.5 \%$ NoMR, $p=0.007)$ and experienced significantly longer postoperative lengths of stay and higher rates of postoperative respiratory failure.

Kaplan-Meier analysis of survival in the overall cohort (Fig. 1A) showed significantly worse medium- and long-term survival for patients in Group MR (log rank $p<0.001)$. In addition, follow-up echocardiograms showed more severe MR at 1 year in Group MR $(1.1 \pm 0.7$ MR vs. $0.6 \pm 0.7$ NoMR, $p<0.001)$ compared to Group NoMR, although the mean severity of MR in both groups fell in the trace-to-mild range. 
Table 1 Overall Cohort Analysis

\begin{tabular}{|c|c|c|c|}
\hline & NoMR & MR & $p$-value \\
\hline \multicolumn{4}{|l|}{ Demographics } \\
\hline Total, n & 1826 & 330 & - \\
\hline Age, years (mean $\pm S D$ ) & $69.3 \pm 14.5$ & $78.1 \pm 10.1$ & $<0.001$ \\
\hline Male, n (\%) & $1061(58.1)$ & $174(52.7)$ & 0.07 \\
\hline $\mathrm{BMl}, \mathrm{kg} / \mathrm{m}^{2}($ mean $\pm \mathrm{SD})$ & $28.0 \pm 5.8$ & $27.2 \pm 5.6$ & 0.03 \\
\hline \multicolumn{4}{|l|}{ Co-morbidities, n (\%) } \\
\hline Myocardial infarction & $156(8.5)$ & $66(20.0)$ & $<0.001$ \\
\hline Congestive heart failure & $329(18.0)$ & $111(33.6)$ & $<0.001$ \\
\hline Severe CKD & $81(4.4)$ & $39(11.8)$ & $<0.001$ \\
\hline Diabetes & $361(19.8)$ & $84(25.5)$ & 0.02 \\
\hline Dialysis & $26(1.4)$ & $9(2.7)$ & 0.09 \\
\hline \multicolumn{4}{|l|}{ Baseline echocardiography } \\
\hline LVEF, \% (mean \pm SD) & $52.1 \pm 12.0$ & $46.1 \pm 14.2$ & $<0.001$ \\
\hline $3-4+\mathrm{Al}, \mathrm{n}(\%)$ & $424(23.2)$ & $65(19.7)$ & 0.16 \\
\hline Severe AS, n (\%) & $1472(80.6)$ & $287(87.0)$ & 0.006 \\
\hline 3-4+ TR, n (\%) & $16(0.9)$ & $11(3.3)$ & $<0.001$ \\
\hline \multicolumn{4}{|l|}{ Operative details } \\
\hline Re-operations, n (\%) & $324(17.7)$ & $96(29.1)$ & $<0.001$ \\
\hline CPB time, minutes (mean $\pm S D$ ) & $88.9 \pm 32.0$ & $92.5 \pm 33.2$ & 0.06 \\
\hline $\mathrm{XCL}$ time, minutes (mean $\pm \mathrm{SD}$ ) & $62.3 \pm 19.8$ & $63.3 \pm 18.8$ & 0.39 \\
\hline \multicolumn{4}{|l|}{ Outcomes } \\
\hline 30-day mortality, n (\%) & $27(1.5)$ & $12(3.6)$ & 0.007 \\
\hline Post-op LOS, days (median, IQR) & $7,5-9$ & $8,6-11$ & $<0.001$ \\
\hline Re-op for bleeding & $60(3.3)$ & $12(3.6)$ & 0.74 \\
\hline Respiratory failure, n (\%) & $91(5.0)$ & $26(7.9)$ & 0.03 \\
\hline New need for dialysis, n (\%) & $18(1.0)$ & $3(0.9)$ & 0.90 \\
\hline \multicolumn{4}{|l|}{ Echocardiographic follow-up } \\
\hline 1-year MR grade (mean \pm SD) & $0.6 \pm 0.7$ & $1.1 \pm 0.7$ & $<0.001$ \\
\hline
\end{tabular}

Abbreviations: $\mathrm{Al}=$ aortic insufficiency, $\mathrm{AS}=$ aortic stenosis, $\mathrm{BMI}=$ body mass index, $\mathrm{CKD}=$ chronic kidney disease, $\mathrm{CPB}=$ cardiopulmonary bypass, $\mathrm{IQR}=$ interquartile range, $L O S=$ length of stay, $L V E F=$ left ventricular ejection fraction, $\mathrm{MR}=$ mitral regurgitation, $\mathrm{TR}=$ tricuspid regurgitation $\mathrm{XCL}=$ aortic cross-clamp

\section{Propensity-matched analysis}

In view of the significant baseline differences between the groups MR and NoMR, a propensity-matched analysis was performed. A total of 330 patients were identified in each group following propensity matching. Baseline characteristics are shown in Table 2. Mean age was $77.9 \pm 10.0$ years and $50.6 \%$ of patients were male. There were no significant differences in co-morbidities. Propensity-matched preoperative echocardiographic data is presented in Table 3. There was no difference in preoperative ejection fraction or prevalence of severe AI, severe AS, or severe TR. Propensity-matched operative details are presented in Table 4. There was no difference in patients undergoing re-operative sternotomy or a

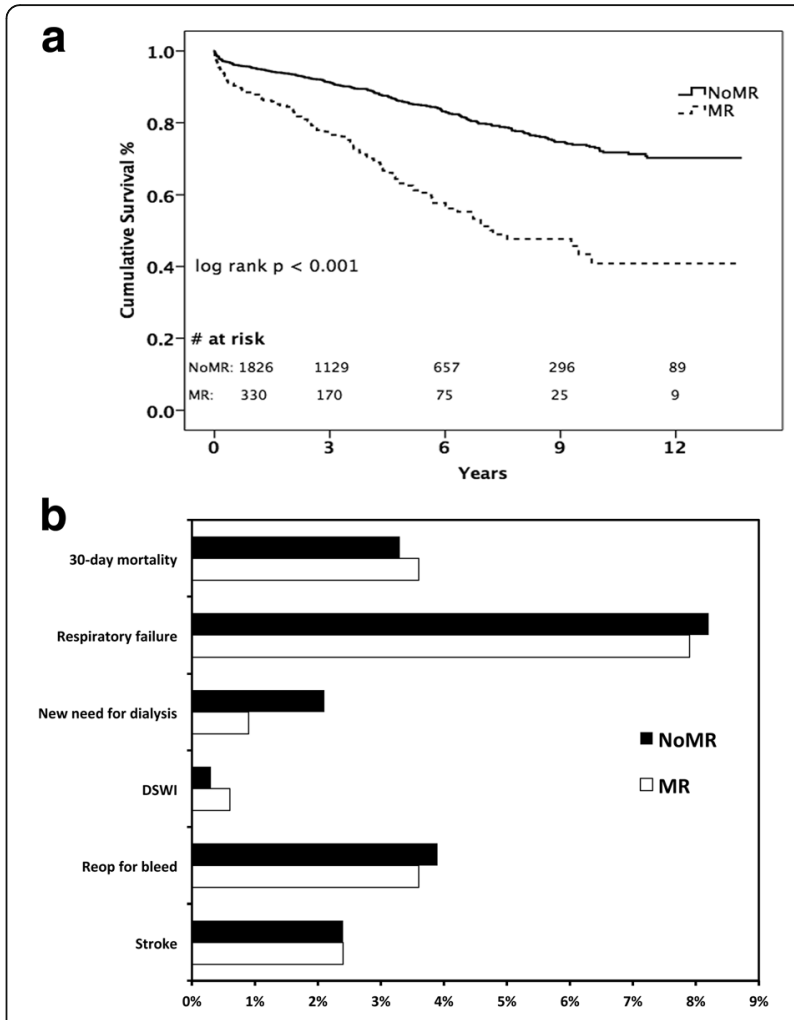

C

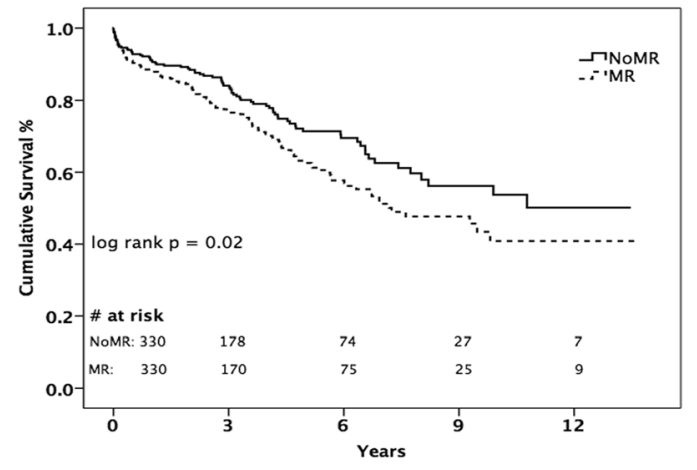

Fig. 1 a Kaplan-Meier analysis of long-term survival in overall cohort stratified by treatment group, (b) Postoperative complications of propensity-matched cohort by treatment group, (c) Kaplan-Meier analysis of long-term survival in propensity-matched cohort by treatment group. (Abbreviations: DSWI = deep sternal wound infection)

minimally invasive approach. The vast majority of patients $(n=298(90.3 \%)$ in MR vs. $n=299(90.6 \%)$ in NoMR, $p=0.9)$ underwent bioprosthetic AVR with no differences between groups.

Propensity-matched postoperative complications and perioperative mortality are shown in Fig. 1B. There was no difference in 30-day mortality between groups $(3.6 \%$ MR vs. $3.3 \%$ NoMR, $p=0.83$ ). Additionally, there were no differences in postoperative complication rates or post-operative length of stay [8 (IQR 6-11) days MR vs. 8 (IQR 6-12) days NoMR, $p=0.47$ ). Kaplan-Meier 
Table 2 Propensity-Matched Baseline Characteristics

\begin{tabular}{llll}
\hline & NoMR & MR & $p$-value \\
\hline Demographics & & & \\
Total, $n$ & 330 & 330 & - \\
Age, years (mean \pm SD) & $77.7 \pm 10.0$ & $78.1 \pm 10.1$ & 0.66 \\
Male, $\mathrm{n}(\%)$ & $160(48.5)$ & $174(52.7)$ & 0.28 \\
BMl, kg/m ${ }^{2}$ (mean \pm SD) & $27.2 \pm 5.8$ & $27.2 \pm 5.7$ & 0.99 \\
Co-morbidities, $n$ (\%) & & & \\
Myocardial infarction & $64(19.4)$ & $66(20.2)$ & 0.85 \\
Congestive heart failure & $111(33.6)$ & $111(33.6)$ & 1.00 \\
Cerebrovascular disease & $33(10.0)$ & $43(13.0)$ & 0.22 \\
Severe CKD & $33(10.0)$ & $39(11.8)$ & 0.45 \\
Peripheral vascular disease & $31(9.4)$ & $31(9.4)$ & 1.00 \\
COPD & $46(13.9)$ & $45(13.6)$ & 0.91 \\
Diabetes & $84(25.5)$ & $66(25.5)$ & 1.00 \\
Dialysis & $9(2.7)$ & $9(2.7)$ & 1.00 \\
\hline
\end{tabular}

Abbreviations: $\mathrm{BMI}=$ body mass index, $\mathrm{CKD}=$ chronic kidney disease, $\mathrm{COPD}=$ chronic obstructive pulmonary disease

Table 3 Propensity-Matched Baseline Echocardiographic Data

\begin{tabular}{|c|c|c|c|}
\hline & NoMR & MR & $p$-value \\
\hline \multicolumn{4}{|l|}{ Ejection fraction } \\
\hline LVEF, \% (mean \pm SD) & $46.7 \pm 14.7$ & $46.1 \pm 14.2$ & 0.61 \\
\hline LVEF > 50\%, n (\%) & $162(50.9)$ & $158(47.9)$ & 0.76 \\
\hline LVEF $30-50 \%, n(\%)$ & $124(37.6)$ & $128(38.8)$ & 0.75 \\
\hline LVEF < 30\%, n (\%) & $44(13.3)$ & $44(13.3)$ & 1.00 \\
\hline \multicolumn{4}{|l|}{ Aortic insufficiency, n (\%) } \\
\hline None/Trace (0) & $136(41.2)$ & $128(38.8)$ & 0.52 \\
\hline Mild (1+) & $92(27.9)$ & $76(23.0)$ & 0.15 \\
\hline Moderate $(2+)$ & $47(14.2)$ & $61(18.5)$ & 0.14 \\
\hline Moderately-Severe/Severe (3-4+) & $55(16.7)$ & $65(19.7)$ & 0.31 \\
\hline \multicolumn{4}{|l|}{ Aortic stenosis, n (\%) } \\
\hline None & $33(10.0)$ & $34(10.3)$ & 0.90 \\
\hline Mild & $1(0.3)$ & $2(0.6)$ & 0.56 \\
\hline Moderate & $3(0.9)$ & $7(2.1)$ & 0.20 \\
\hline Severe & $293(88.8)$ & $287(87.0)$ & 0.47 \\
\hline \multicolumn{4}{|l|}{ Mitral regurgitation, n (\%) } \\
\hline None/Trace & $172(52.1)$ & $0(0)$ & $<0.001$ \\
\hline Mild & $158(47.9)$ & $0(0)$ & $<0.001$ \\
\hline Moderate/Moderately-Severe & $0(0)$ & $330(100)$ & $<0.001$ \\
\hline \multicolumn{4}{|l|}{ Tricuspid regurgitation } \\
\hline None & $217(65.8)$ & $216(65.5)$ & 0.94 \\
\hline Mild & $69(20.9)$ & $66(20.0)$ & 0.77 \\
\hline Moderate & $34(10.3)$ & $37(11.2)$ & 0.71 \\
\hline Severe & $10(3.0)$ & $11(3.3)$ & 0.82 \\
\hline
\end{tabular}

Abbreviations: LVEF = left ventricular ejection fraction
Table 4 Propensity-Matched Operative Characteristics

\begin{tabular}{llll}
\hline & NoMR & MR & $p$-value \\
\hline Re-operation, $\mathrm{n}(\%)$ & $105(31.8)$ & $96(29.1)$ & 0.45 \\
Minimally invasive approach, $\mathrm{n}(\%)$ & $17(5.2)$ & $15(4.5)$ & 0.72 \\
CPB time, minutes (mean \pm SD) & $89.5 \pm 26.6$ & $92.5 \pm 33.2$ & 0.19 \\
XCL time, minutes (mean \pm SD) & $61.9 \pm 18.2$ & $63.3 \pm 18.8$ & 0.36 \\
Prosthesis type, n (\%) & & & \\
$\quad$ Biological & $299(90.6)$ & $298(90.3)$ & 0.90 \\
$\quad$ Mechanical & $26(7.9)$ & $26(7.9)$ & 1.00 \\
$\quad$ Homograft & $5(1.5)$ & $6(1.8)$ & 0.76 \\
\hline
\end{tabular}

Abbreviations: $\mathrm{CPB}=$ cardiopulmonary bypass, $\mathrm{XCL}=$ aortic cross-clamp

survival analysis of the propensity-matched cohort (Fig. 1C) showed significantly worse medium and long-term survival in Group MR (log rank $p=0.02$ ). At 1-year follow-up, mean MR severity was significantly worse in Group MR, although both groups fell in the trace-to-mild range $(1.1 \pm 0.7 \mathrm{MR}$ vs. $0.8 \pm 0.7$ NoMR, $p=0.03$ ).

\section{Discussion}

Concomitant fMR in patients presenting for surgical AVR remains a challenging clinical problem. Although the predominant opinion is that fMR should improve following correction of aortic valve pathology, it is not clear that postoperative relief of left ventricular pressure-volume overload is sufficient to cause significant regression of moderate fMR, which may subsequently limit functional status and postoperative survival. While it seems that mild preoperative fMR will regress following isolated AVR, studies have shown that many patients with moderate preoperative fMR still demonstrate a clinically significant level of postoperative regurgitation on follow-up [6-23]. Given the lack of well-defined treatment guidelines for these patients and small sample sizes used in prior studies, further investigation of the late effects of isolated AVR on moderate fMR is required.

The current study is, to the best of our knowledge, the largest single-center experience and the only propensity-matched analysis in this patient population. We have demonstrated that unaddressed moderate to moderate-severe $(2-3+)$ fMR at the time of isolated AVR leads to equivalent perioperative survival but worsened medium- and long-term survival compared to patients with no or mild preoperative fMR. We did not detect a difference in postoperative complication rates or postoperative length of stay between groups. One-year echocardiographic follow-up revealed that patients with $2-3+$ preoperative fMR had slightly but significantly worse residual MR compared to patients with mild or no preoperative fMR. While the post-AVR left ventricular reverse remodeling may 
improve fMR severity to some degree, our findings show that preoperative $2-3+$ fMR in isolated AVR patients presages poorer late survival.

Review of our overall cohort analysis showed that patients with 2-3+ fMR are sicker than patients with no or mild fMR. Patients in Group MR were significantly older with worse renal and cardiac function, as evidenced by higher rates of congestive heart failure and lower ejection fractions. Group MR patients also had more severe TR, suggesting higher degrees of pulmonary hypertension and right ventricular dysfunction. Several prior studies addressing this population demonstrated similar findings $[9,11,14]$. Although it is possible that the worse prognosis of Group MR patients in the overall analysis is a direct effect of a greater degree of fMR, it is more likely that these patients simply have more advanced cardiovascular disease resulting in worsened overall survival. While our propensity-matched analysis allowed us to control for some of these baseline differences to more specifically evaluate the effect of preoperative fMR, it is worth noting that patients presenting with $2-3+\mathrm{fMR}$ in the setting of aortic valve disease generally have more advanced cardiac disease than their counterparts with no or mild fMR, and should be treated accordingly.

While many studies have demonstrated that severe MR should be repaired during concomitant AVR, controversy remains concerning the correct management of moderate and moderate-severe MR [2-4, 19-24]. Late survival in prior studies comparing patients with $2-3+$ preoperative MR to patients with no or mild MR has varied. Given that the question at hand is whether or not isolated AVR is sufficient treatment for these patients, those studies that found a survival difference generally concluded that mitral intervention should be considered in patients with moderate or greater preoperative MR [6, 7, 13, 19-23]. However, several other studies, including the largest single-center study from the Mayo Clinic $(n=190)$, found no difference in survival between the two groups, suggesting that mitral intervention may be unnecessary $[8,11]$. Nonetheless, large baseline demographic differences among groups are present in these studies, fundamentally confounding interpretation of the data. Given the clinical equipoise in the conclusions of prior studies and the potential that moderate fMR may simply be an indicator of more advanced disease rather than a causative entity leading to poorer late survival, a propensity-matched analysis was essential to control for key baseline differences and remove confounding comorbidities. After propensity-matching, our data suggest that a lower bar for mitral intervention in these patients may be warranted. Further study into specific patient subgroups may be necessary to clarify the tradeoff between additional perioperative risk and late mortality with combined aortic and mitral surgery.

Our data suggest that patients with moderate, concomitant fMR undergoing AVR would benefit from mitral intervention, although the logical follow-up question to that observation is to ask whether mitral valve repair or replacement would confer the lowest perioperative mortality and the highest late survival in this population. A Cardiothoracic Surgery Trials Network study from Acker and colleagues evaluated the performance of mitral valve repair vs. replacement for patients with ischemic MR not undergoing AVR and found no difference in perioperative or 1-year survival, but the recurrence rate of moderate or severe MR was higher in the repair group [25]. Although not directly applicable to patients with combined aortic and mitral valve disease, follow-up of this randomized trial could help to predict which mitral intervention confers a greater survival benefit to patients.

Few studies exist that directly address AVR/mitral repair vs. AVR/mitral replacement in the $\mathrm{fMR}$ subgroup. In 2003, the Cleveland Clinic group published a study addressing all patients who underwent combined AVR with either mitral valve repair or replacement for any reason, not solely for fMR [26]. While there was no difference in perioperative survival between mitral repair and replacement, they found that concomitant mitral repair with AVR resulted in significantly improved survival at 5, 10, and 15-year follow-up compared to mitral valve replacement with AVR [26]. However, only $7 \%$ of the total population had fMR in the Cleveland Clinic series, which again highlights the need for a large randomized trial evaluating the appropriate therapy for patients with aortic valve disease and significant concomitant fMR [26].

There are several limitations to our study. It is a retrospective, single-center study that reflects the treatment biases of our clinical team. We have attempted to limit preoperative baseline differences by propensity matching, but it is possible that our propensity-matched groups do not accurately reflect the true population based on unmeasured covariates. Follow-up survival information did not include the cause of late death, so all comparisons of mortality reflect all-cause mortality and not death from cardiovascular causes. Finally, formal quantification of MR using more advanced echocardiographic parameters, such PISA derived metrics, will be necessary in future studies; these measurements are now a standard part of our echocardiographic analysis, but were not available for the majority of this patient dataset during our study period. 


\section{Conclusions}

In conclusion, patients undergoing isolated AVR with concomitant moderate and moderate-severe preoperative fMR have worse medium- and long-term survival, and continue to have elevated MR severity at 1 year, compared to patients with no or mild preoperative fMR. These results indicate the need for mitral valve intervention at the time of AVR in patients with moderate or greater fMR. Given our results and those from prior studies, a randomized trial is needed to definitively clarify the optimal treatment strategy for this population, and to determine whether mitral valve repair or replacement at the time of AVR for moderate or greater preoperative $\mathrm{fMR}$ is superior.

\section{Abbreviations}

Al: aortic insufficiency; AS: aortic stenosis; AVR: aortic valve replacement; fMR: functional mitral regurgitation; TR: tricuspid regurgitation

\section{Availability of data and materials}

The datasets used are available from the corresponding author on reasonable request.

\section{Authors' contributions}

$\mathrm{RS}, \mathrm{AO}, \mathrm{HY}, \mathrm{DH}, \mathrm{MAA}, \mathrm{MA}, \mathrm{CRS}$, IG all contributed to data collection, study idea, and writing and revisions of the manuscript. All authors read and approved the final manuscript.

\section{Ethics approval and consent to participate}

The study was approved by the Columbia University Institutional Review Board and need for individual patient consent was waived.

\section{Consent for publication}

Not applicable.

\section{Competing interests}

Isaac George is a consultant for Medtronic and for Edwards Lifesciences Inc Craig Smith is the Surgical Principal Investigator of the PARTNER and PARTNER II Trials, for which travel and customary expenses associated with Trial management are reimbursed by the sponsor, Edwards LifeSciences.

\section{Publisher's Note}

Springer Nature remains neutral with regard to jurisdictional claims in published maps and institutional affiliations.

Received: 29 March 2018 Accepted: 7 June 2018

Published online: 19 June 2018

\section{References}

1. Society for Thoracic Surgery Adult Cardiac Surgery Database, 2014 Harvest 1-Exectuive summary, 2014.

2. Nishimura RA, Otto CM, Bonow RO, Carabello BA, Erwin JP, Guyton RA, et al. 2014 AHAVACC guideline for the Management of Patients with Valvular Heart Disease: a report of the American College of Cardiology/American Heart Association task force on practice guidelines. Circulation. 2014;129(23):2440-92.

3. Nishimura RA, Otto CM, Bonow RO, Carabello BA, Erwin JP, Fleisher LA, et al. 2017 AHA/ACC focused update of the 2014 AHA/ACC guideline for the management of patients with valvular heart disease: a report of the American College of Cardiology/American Heart Association task force on clinical practice guidelines. Circulation. 2017;135(25):e1159-95.

4. Bonow RO, Brown AS, Gillam LD, Kapadia SR, Kavinsky CJ, Lindman BR, et al. ACC/AATS/AHA/ASE/EACTS/HVS/SCA/SCAI/SCCT/SCMR/STS 2017 appropriate use criteria for the treatment of patients with severe aortic stenosis: a report of the American College of Cardiology Appropriate use Criteria Task Force, American Association for Thoracic Surgery, American Heart Association, American Society of Echocardiography, European
Association for Cardio-Thoracic Surgery, heart valve society, Society of Cardiovascular Anesthesiologists, Society for Cardiovascular Angiography and Interventions. J Am Soc Echocardiogr. 2018;31(2):117-47.

5. Come P, Riley M, Ferguson JF, Morgan JP, McKay RG. Prediction of severity of aortic stenosis: accuracy of multiple noninvasive parameters. Am J Med. 1988:85:29-37.

6. Barreiro CJ, Patel ND, Fitton TP, Williams JA, Bonde PN, Chan V et al. Aortic Valve Replacement and Concomitant Mitral Valve Regurgitation in the Elderly: Impact on Survival and Functional Outcome. Circulation 2005; 112(s1):I-443-1-447

7. Harling L, Saso S, Jarral OA, Kourliouros A, Kidher E, Athanasiou T. Aortic valve replacement for aortic stenosis in patients with concomitant mitra regurgitation: should the mitral valve be dealt with? Eur J Cardiothorac Surg. 2011:40:1087-96

8. Absil B, Dagenais F, Mathieu P, Metras J, Perron J, Baillot R, et al. Does moderate mitral regurgitation impact early or mid-term clinical outcome in patients undergoing isolated aortic valve replacement for aortic stenosis? Eur J Cardiothorac Surg. 2003;24:217-22.

9. Takeda K, Matsumiya G, Sakaguchi T, Miyagawa S, Yamauchi T, Shudo Y, et al. Impact of untreated mild-to-moderate mitral regurgitation at the time of isolated aortic valve replacement on late adverse outcomes. Eur J Cardiothorac Surg. 2010;37:1033-8.

10. Lim JY, Jung SH, Kim JB, Chung CH, Lee JW, Song H. Management of concomitant mild to moderate functional mitral regurgitation during aortic valve surgery for severe aortic insufficiency. J Thorac Cardiovasc Surg. 2014; 148(2):441-6.

11. Wan CKN, Suri RM, Li Z, Orszulak TA, Daly RC, Schaff HV, et al. Management of moderate functional mitral regurgitation at the time of aortic valve replacement: is concomitant mitral valve repair necessary. J Thorac Cardiovasc Surg. 2009;137(3):635-40.

12. Ruel M, Kapila V, Price J, Kulik A, Burwash IG, Mesana TG. Natural history and predictors of outcome in patients with concomitant functional mitral regurgitation at the time of aortic valve replacement. Circulation. 2006; 114(s1):I-541-6.

13. Moazami N, Diodato MD, Moon MR, Lawton JS, Pasque MK, Herren RL, et al. Does functional mitral regurgitation improve with isolated aortic valve replacement? J Card Surg. 2004;19:444-8.

14. Caballero-Borrego J, Gomez-Doblas JJ, Cabrera-Bueno F, García-Pinilla JM, Melero JM, Porras C, et al. Incidence, associated factors and evolution of non-severe functional mitral regurgitation in patients with severe aortic stenosis undergoing aortic valve replacement. Eur J Cardiothorac Surg. 2008;34:62-6.

15. Brasch AV, Khan SS, DeRobertis MA, Kong JH, Chiu J, Siegal RJ. Change in mitral regurgitation severity after aortic valve replacement for aortic stenosis. Am J Cardiol. 2000:85:1271-4.

16. Alghamdi AA, Elmistekawy EM, Singh SK, Latter DA. Is concomitant surgery for moderate functional mitral regurgitation indicated during aortic valve replacement for aortic stenosis? A systematic review and evidence-based recommendations. J Card Surg. 2010:25(2):182-7.

17. Waisbren EC, Stevens LM, Avery EG, Picard MH, Vlahakes GJ, Agnihotri AK. Changes in mitral regurgitation after replacement of the stenotic aortic valve. Ann Thorac Surg. 2008;86(1):56-62.

18. Kowalówka AR, Onyszczuk M, Wańha W, Deja MA. Do we have to operate on moderate functional mitral regurgitation during aortic valve replacement for aortic stenosis? Interact Cardiovasc Thorac Surg. 2016;23(5):806-9.

19. Jeong DS, Park PW, Sung K, Kim WS, Yang JH, Jun TG, et al. Long-term clinical impact of functional mitral regurgitation after aortic valve replacement. Ann Thorac Surg. 2011;92(4):1339-45.

20. Joo HC, Chang BC, Cho SH, Youn YN, Yoo KJ, Lee S. Fate of functional mitral regurgitation and predictors of persistent mitral regurgitation after isolated aortic valve replacement. Ann Thorac Surg. 2011;92(1):82-7.

21. Matsumura Y, Gillinov AM, Toyono M, Oe H, Yamano T, Takasaki K, et al. Echocardiographic predictors for persistent functional mitral regurgitation after aortic valve replacement in patients with aortic valve stenosis. Am J Cardiol. 2010;106(5):701-6.

22. Unger P, Dedobbeleer C, Van Camp G, Plein D, Cosyns B, Lancellotti P. Mitral regurgitation in patients with aortic stenosis undergoing valve replacement. Heart. 2010:96(1):9-14.

23. Schubert SA, Yarboro LT, Madala S, Ayunipudi K, Kron IL, Kern JA, et al. Natural history of coexistent mitral regurgitation after aortic valve replacement. J Thorac Cardiovasc Surg. 2016;151(4):1032-42. 
24. Eynden FV, Bouchard D, El-Hamamsy I, Butnaru A, Demers P, Carrier M, et al. Effect of aortic valve replacement for aortic stenosis on severity of mitral regurgitation. Ann Thorac Surg. 2007:83(4):1279-84.

25. Acker MA, Parides MK, Perrault LP, Moskowitz AJ, Gelijns AC, Voisine P, et al. Mitral-valve repair versus replacement for severe ischemic mitral regurgitation. New Engl J Med. 2014;370:23-32.

26. Gillinov AM, Blackstone EH, Cosgrove DM, White J, Kerr P, Marullo A, et al. Mitral valve repair with aortic valve replacement is superior to double valve replacement. J Thorac Cardiovasc Surg. 2003;125:1372-87.

- fast, convenient online submission

- thorough peer review by experienced researchers in your field

- rapid publication on acceptance

- support for research data, including large and complex data types

- gold Open Access which fosters wider collaboration and increased citations

- maximum visibility for your research: over $100 \mathrm{M}$ website views per year 